Jason Bazil, Purdue University

Aaron Kyle, Purdue University

Suneera Bhatia, Purdue University

Brain Moerdyk, Purdue University

Thomas Talavage, Purdue University

Allison Sieving, Purdue University

Andrew Brightman, Purdue University

George Graber, Purdue University

Ann Rundell, Purdue University 


\title{
Bioinstrumentation Instruction through Hybrid Wet/Circuit Laboratory Activities
}

\begin{abstract}
Biomedical Engineering undergraduate programs traditionally consist of a linear circuits course preceding a Bioinstrumentation course. Recently, many undergraduate programs have replaced this sequence with only a measurement principles course that does not require a background in circuit theory. Unfortunately, this consolidation leaves a deficit in the students' understanding of basic electrical principles. To address these issues, the Weldon School of Biomedical Engineering at Purdue University has developed a unique, one semester, two credit junior-level course covering bioinstrumentation, bioelectricity, and measurement principles while only requiring previous courses in differential equations and basic physics. The class consists of a weekly three hour laboratory supplemented by a 50 minute lecture. The lecture portion of this course briefly presents the underlying circuit and theoretical concepts underpinning the laboratory procedure. It is anticipated that the inquiry-based and hands-on laboratory exercises in conjunction with extensive pre- and post-lab assignments teach complex bioinstrumentation, bioelectricity and measurement concepts. Basic circuit design and analysis are incorporated into 13 hybrid wet/circuit labs, ensuring that all circuit and signal topics are explored within the context of a biomedical phenomenon such as axonal membrane models, impedance pneumography, the basilar membrane of the cochlea, and the electrocardiogram. These weekly laboratories and pre- and post-lab exercises help to develop problem solving skills, critical analysis, independent study and life long learning skills. The laboratory component culminates in a two-week design project to solve a bioinstrumentation problem. Students employ a structured design process in problem formulation, brainstorming, research, design evaluation, and implementation. After the initial offering of this course, students learned basic circuit theory and fundamental measurement principles. These laboratory exercises develop the students' understanding of bioelectric phenomena and bioinstrumentation, and their ability to accurately measure physiological events.
\end{abstract}

\section{Introduction}

Many Biomedical Engineering (BME) programs have implemented a bioinstrumentation course requiring prior linear circuit theory instruction. Such programs often evolved from Electrical Engineering (EE) classes that had been re-configured for BME. This provided the students with a basic understanding of EE concepts, enabling the students to apply circuit concepts to bioinstrumentation. Conversely, some BME departments cover bioinstrumentation through a measurements principles course, neglecting the underlying circuit principles. This approach teaches students how to use measurement devices, but misses an opportunity to expose students to the operating principles of these measurement devices and engineering analysis techniques such as system modeling and time and frequency domain analysis.

To incorporate EE principles into a BME curriculum, the Weldon School of Biomedical Engineering has developed a novel, one semester bioinstrumentation laboratory course. The prerequisites for this course are differential equations and basic engineering physics courses. A weekly 50 minute lecture introduces students to relevant circuit and engineering principles while the students apply the concepts and explore their relation to biological systems and physiological measurements during the accompanying 3 hour laboratory. Rather than have students 
compartmentalize topics, the course activities are structured to promote coalescence of biological phenomena and electrical engineering models and concepts. By presenting the students with authentic problems incorporated into each post-lab assignment and the design project, the course facilitates students to develop adaptive knowledge and enables them to better perform as biomedical engineers. 'This report provides a detailed description of the course's development, an overview of several of the laboratory exercises, and a qualitative assessment of the course effectiveness.

\section{Laboratory Course Development}

The inception of this course stemmed from a series of faculty workshops to design Purdue's undergraduate BME curriculum. As with many BME programs across the country, our program had to address the heavy course load demand on the students' time. This laboratory course was envisioned as a means to teach several fundamental BME principles with minimal lecture time. It provides the students with an environment for hands-on, inquiry-based, and active learning of bioinstrumentation, bioelectricity, and measurement principles.

A team composed of two BME faculty members, four part-time developmental teaching assistants (TA), and three engineering staff met weekly to develop the course from its mission statement to its current form. The faculty were responsible for the lab content and concept delivery. TAs developed the detailed lab procedures and drafted lab manual. The engineering staff assisted with laboratory implementation details, designed and built specialized lab tools, purchased laboratory equipment, and prepared the final version of the laboratory manual. The development process took roughly one year with approximately 20 hours per week of TA developmental time.

Efforts were made to ensure that the laboratory was not simply an EE circuits laboratory, but instead taught engineering concepts through activities that were related to bioelectricity, electrochemistry or physiological measurements. Weekly course development meetings often contained brainstorming sessions to determine how to best address a technical concept from a BME perspective. This process was often iterative, with the developmental TAs testing proposed procedures for feasibility, repeatability, and, most importantly, concept demonstration. The laboratory activities were intentionally varied in nature so that students were exposed to different learning methods. Some activities had students design their own procedures to accomplish a goal; others had students implement both circuit and wet-lab models of the same concept to assist with the translational learning. Data analysis and data presentation were left open-ended, such that the students had to decide which presentation method would be most effective.

\section{Laboratory Course Operation}

The laboratory environment supporting this course requires standard electrical instrumentation such as voltmeters, ammeters, oscilloscopes, power supplies, and more specialized equipment including conductive cells, impedance pneumographs, electrocardiogram (ECG) preamplifiers, audiometers, isolated stimulating units, etc. Each lab group purchased the lab manual, a circuit board (breadboard), and a component kit containing standard resistors, capacitors, inductors, and op amps. 
The course was first offered as a pilot lab during the Summer 2005 session to three undergraduate students from the School of Electrical and Computer Engineering and one student from Interdisciplinary Engineering. These pilot students were deliberately selected since they have basic circuits knowledge with limited biology background. This approach helped the course development group debug the lab procedures from the circuit perspective while testing the ability of the laboratory activities to teach bioelectricity and measurement principles. From the feedback from the pilot students and instructor evaluations, improvements were made to the laboratory activities and course manual. Thirty-six BME juniors completed the course during the Fall 2005 semester.

Students were required to maintain a standard engineering notebook during laboratory activities. Each student's notebook included circuit schematics, hypotheses, and experimental data. Each procedure contained extensive pre- and post-lab activities aimed at reviewing the lab and lecture concepts. Post-lab assignments contained textbook problems, experiment analysis questions, and computer programming problems (typically using MATLAB, MathCAD, or PSPICE). Each post-lab assignment also proposed a related design question, which required students to critically analyze a realistic problem and generate a potential solution.

In addition to graded lab reports, students' comprehension was quantified throughout the semester via three exams and a laboratory practical. Midterm and final examinations were used to gauge the students' abilities to solve circuit problems and interpret recorded data. A laboratory practical exam evaluated the students' ability to properly construct a circuit from a schematic, design measurement experiments, and utilize electrical laboratory equipment.

\section{Laboratory Course Content}

The learning objectives set forth for this course state that upon completion of the course each student will be able to:

1. Employ circuit models and analysis techniques to understand, interpret, and predict bioelectric system behaviors

2. Conduct, observe, and document laboratory experiments to test hypotheses and predictions

3. Design bioinstrumentation systems with adequate bandwidth, amplitude linearity, and phase linearity to faithfully record a physiological event

A total of 13 hybrid wet/circuit laboratories and a two week design lab are used to achieve these objectives. The experiments, the associated engineering, and their related biological or wet components are displayed in Table 1. 


\section{Lab Procedure}

Wet/Biological Component

(Engineering Concepts)

\begin{tabular}{|c|c|c|c|c|c|c|c|c|}
\hline $\begin{array}{l}\text { Fundamentals of electrical measurements and properties } \\
\text { (Basic circuit concepts and Ohm's Law) }\end{array}$ & $\mathrm{X}$ & & & & & & $\mathrm{X}$ & \\
\hline $\begin{array}{l}\text { Resistive models of biological systems } \\
\text { (KCL, KVL, and Nodal Analysis) }\end{array}$ & & & & & & & & \\
\hline $\begin{array}{l}\text { Generation and measurement of bioelectric signals } \\
\text { (Linearity and Thevenin equivalents) }\end{array}$ & & $\mathrm{X}$ & $\mathrm{X}$ & & & & & \\
\hline $\begin{array}{l}\text { Time varying bioelectric signals and response times of measurement devices } \\
\text { (Capacitors, inductors, step response for } R C \text { and } R L \text { circuits) }\end{array}$ & & & & $\mathrm{X}$ & & & $\mathrm{X}$ & \\
\hline $\begin{array}{l}\text { Bioelectric amplifiers } \\
\text { (Operational amplifiers) }\end{array}$ & & & X & & X & & & \\
\hline $\begin{array}{l}\text { Frequency response of RC models of biological systems } \\
\text { (Simusoids and simusoidal steady state response) }\end{array}$ & X & & & & & & & \\
\hline $\begin{array}{l}\text { Adequate bandwidth for measurement devices } \\
\text { (Phasors with circuit analysis) }\end{array}$ & & & & X & & & & \\
\hline $\begin{array}{l}\text { The RLC Model of the Cochlea } \\
\text { (RLC circuits) }\end{array}$ & & & & & & $\mathrm{X}$ & & \\
\hline $\begin{array}{l}\text { Recording and stimulating electrodes } \\
\text { (Electrode RC models) }\end{array}$ & X & & & & & & X & \\
\hline $\begin{array}{l}\text { Stimulation of nervous tissue } \\
\text { (Transfer functions and convolution) }\end{array}$ & & X & X & & & & X & \\
\hline $\begin{array}{l}\text { Signal propagation in nervous tissue } \\
\text { (Laplace Transforms) }\end{array}$ & & & X & & & & X & \\
\hline $\begin{array}{l}\text { Instrumentation amplifiers } \\
\text { (Advanced op-amp circuits) }\end{array}$ & & & & $\mathrm{X}$ & & & & \\
\hline $\begin{array}{l}\text { Design project } \\
\text { (Crrcuit analysis in s-domain and criteria for faithful reproduction of } \\
\text { physiological signals) }\end{array}$ & & & & & & & & $\mathrm{X}$ \\
\hline
\end{tabular}

Table 1. Laboratory activities are listed with engineering concepts in italics with the corresponding wet/biological components indicated with an "X.".

\subsection{Overview of Labs}

The laboratories can be broadly classified as circuit analysis, amplifiers, time and frequency domain analysis, or living subject measurement experiments. The first four laboratories develop the students' knowledge of electrical circuits and the governing principles necessary to analyze these circuits. Students investigate simple resistive models of biological systems as well as electrical methods to monitor physiological events. Students are also introduced in these labs to resistor-capacitor (RC) circuits and response times of measurement devices. The measurement device concepts are relevant for the entire course as these devices are consistently used to measure signals of interest. The experiments demonstrate how bioelectric signals, such as an action potential traveling down an axon, can be modeled using an electric circuit. Also presented are measurement principles that emphasize how resistive and capacitive components of the measurement device input impedances can lead to signal distortion.

Several labs focus on operational amplifiers and their importance in measurement devices. Amplifiers are discussed in terms of basic amplifier configurations and their use in making physiological signals measurable. Simple differential amplifiers are discussed before moving on to the more complicated instrumentation amplifiers that are commonly used in medical diagnostic devices. The students design and implement these amplifiers to explore the importance of loading, impedance matching, common mode rejection ratio (CMRR), and the dynamic range of an amplifier. 
Four labs primarily address time varying signals, the frequency response of linear systems, and the use of resistive, inductive, and capacitive (RLC) circuit elements to model biological systems. The importance of amplitude linearity, adequate bandwidth, and phase linearity for accurate measurement of biological events are emphasized during these labs. The students build a simplified RLC circuit model of the cochlea to investigate the frequency response of the inner ear, while also examining the behavior of resonant circuits.

The last three labs allow students to obtain accurate measurements of physiological signals from living subjects: North Grass bullfrogs. In these experiments students examine excitability of nervous tissue, derive a strength-duration curve for electrical current intensity necessary to excite a frog's sciatic nerve, and investigate the waveform and nerve propagation velocity (NPV) of an action potential. Students are fully responsible for the preparation of their experimental subject: they perform the dissection, isolate the nerve of interest, and use the necessary circuitry to obtain reliable measurements. During the NPV experiment, the students engage in an exploratory exercise where they must develop a method to quantify the NPV of the sciatic nerve using recording electrodes. The students examine the biphasic nature of an action potential by changing the separation distance between the recording anode and cathode. The final animal subject laboratory involves the design of an instrumentation amplifier to measure the frog's ECG.

\subsection{Descriptions of Exemplar Laboratory Activities}

To illustrate how the bioinstrumentation, bioelectricity and measurement principles are integrated and taught within a BME context for this course, two typical labs and the design project are further described herein.

\subsubsection{Lab Addressing Resistive Models of Biological Systems}

The purpose of this laboratory is to investigate resistive circuits and their analysis techniques and provide an understanding of some basic resistive models of biological systems. The specific learning objectives of this lab are to:

1. Apply circuit analysis techniques to evaluate voltage and current in resistive circuits confirming theoretical computations.

2. Become familiar with a simplified resistive core conductor model of excitable tissue.

3. Understand the origin of electrical safety rules.

4. Investigate the principles underlying impedance pneumography

A simplified resistive core conductor model of excitable tissue represents the resistive impedance encountered by an action potential as it travels down an axon. Students obtain voltage measurements along each branch of the resistive ladder network to determine the voltage drop across each stage. From this activity, the students gain an intuitive understanding of how the bioelectric signal travels along an axon. They also compare their empirical results to prelab predictions made using Kirchoff's current and voltage laws and nodal analysis.

As an illustration of the origin of electrical safety protocols, students construct a resistor-man circuit (Figure 1). The components of the model are weighted to approximately represent resistance of an adult human's limbs, head, neck, and torso. Students measure the voltage across and calculate the current through the circuit elements when a voltage is applied. This exercise 
elucidates the concept of current traveling the path of least resistance to ground and explores the possible damage a person might incur when a current is sent through one of their limbs or torso. This experiment also demonstrates of how biological structures can be modeled as electrical circuit elements.

Respiration can be monitored by observing changes in the thoracic cavity's electrical impedance during inspiration and expiration. A hybrid circuit model is used to illustrate the basic principles underlying this physiological measurement technique. After developing an intuitive understanding using a circuit model, the students utilize impedance pneumography to monitor the respiration of a living subject. The hybrid circuit model measures the voltage across two electrodes in a saline conducting cell with different sized acrylic blocks placed between the electrodes. The block sizes represent breaths of varying depth, thus producing different impedances between the electrodes. Following this activity, the students design and conduct an experiment to explore the measurement of respiration using impedance pneumography with the TA serving as the living subject and utilizing a commercial impedance pneumograph (UFI model RESP $1 /$ EKG).

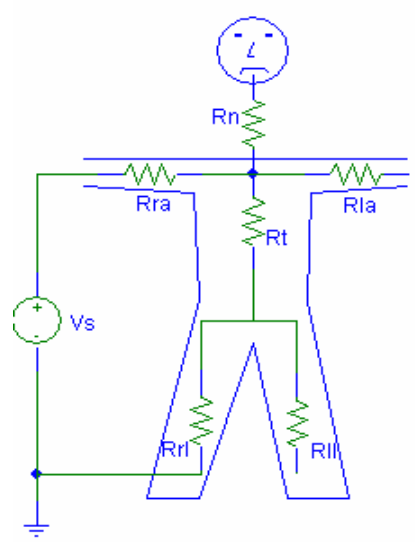

A.

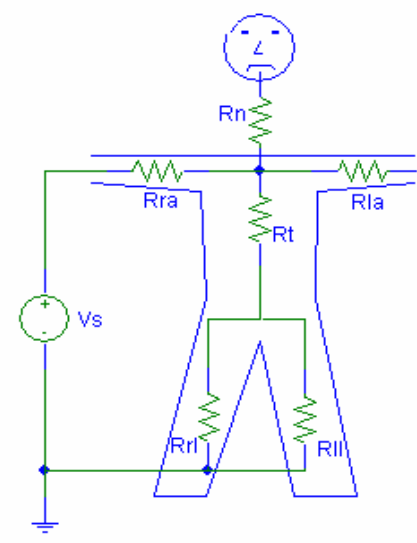

B.

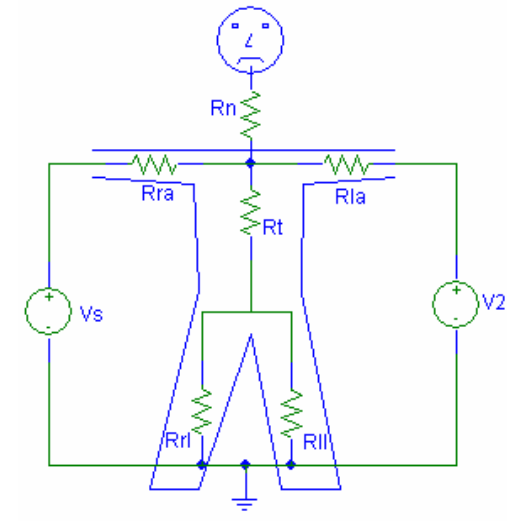

C.

Figure 1. Ready the Resistor Man circuit model. (A) Right arm and foot are connected to the voltage source while other "limbs" float, (B) right arm connected to the positive terminal and both feet are connected to ground, while the other "limbs" float, and (C) a voltage is applied across the chest with both feet grounded.

\subsubsection{Lab Addressing Signal Propagation in Nervous Tissue}

The specific learning objectives of this lab explicitly address bioelectricity and measurement principles. These learning objectives are:

1. Understand and observe how excitable tissue (in particular nervous and muscle tissue) conducts and responds to electrical signals

2. Understand how the observed bioelectric waveform is a function of electrode placement and signal propagation velocity

Students are introduced to the mechanisms by which action potentials (AP) are generated from ion fluxes across a cellular membrane, and techniques for the measurement of these signals. 
They examine how excitable tissue (in particular nervous and muscle tissue) responds to electrical stimuli using a bipolar electrode. The electromyography of the frog's gastrocnemius is measured, and the students investigate bioelectric phenomena such as threshold stimulus for muscular excitation and motor-end plate fatigue. The nerve tissues are examined with regard to NPV and the AP waveform. To quantify the propagation velocity along the sciatic nerve, the students develop and conduct their own experimental technique to determine the NPV. Also, the effect of electrode position on recorded AP waveform morphology is examined using a multichannel bipolar electrode. Assuming the NPV remains constant, students should observe the biphasic nature of the waveform with increasing electrode separation (Figure 2). This lab exercise clearly demonstrates that the observed waveform is a function of electrode placement and signal propagation velocity.

A.

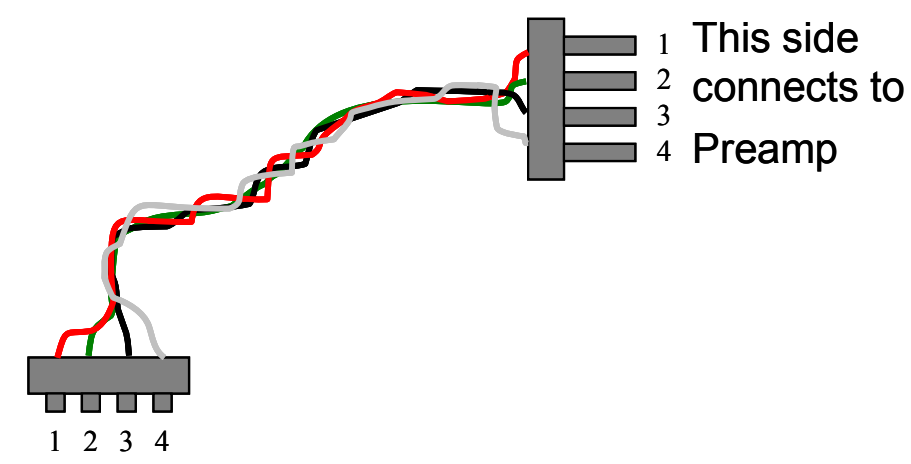

B.
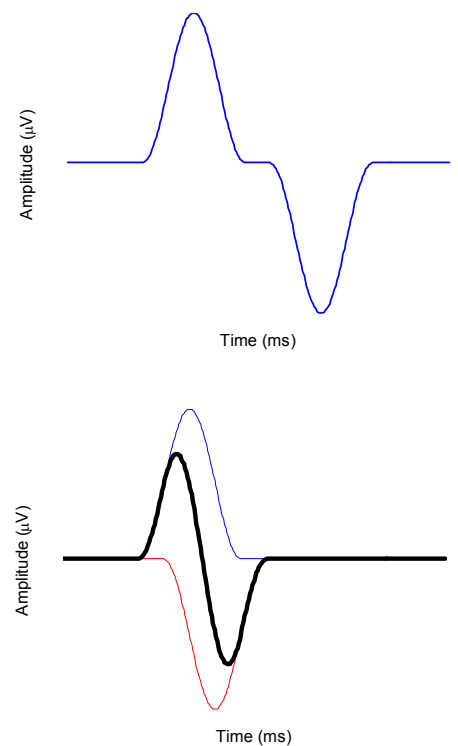

Figure 2. (A) The multi-channel electrode was implemented to examine (B) theoretical schematic of the biphasic AP morphology recorded via the sciatic nerve.

\subsubsection{Design Project}

The laboratory exercises culminate in a two week design project focusing on the development of a medical diagnostic device. The goal of this project is for students to apply concepts developed throughout the semester to the design and implementation of an electronic monitoring device. Students are expected to design the sensing circuitry using the prescribed equipment, develop testing protocols and debug their circuit for optimal performance, demonstrate that device performs within specifications, and submit a professional, formal report on the device. The specific learning objectives of the design project are:

1. Given an engineering problem statement, employ an engineering design process to design a device to meet the specifications.

2. Build, calibrate, and test designed device to ensure it meets the customer's needs.

3. Identify applicable regulatory agencies and specific guidelines.

4. Document the design process and outcome including the considerations given to economics, regulatory issues, and relevant societal impact issues in a formal report. 
The proposed problem for the Fall 2005 semester was to design a hand dynamometer that could be used to measure grip strength and monitor muscle atrophy. The completed dynamometer was required to be portable, self-contained and have an analog voltage display, with an output of $1 \mathrm{~V}$ per $1 \mathrm{~kg}$ of applied force. Students are supplied with a pre-assembled dynamometer unit with attached strain gauge, an analog voltmeter display, and two $9 \mathrm{~V}$ batteries. They are to use parts from their lab component kits to integrate the strain gauge, sensing circuitry, and power sources into a complete measurement system.

Students are expected to develop and implement their design over two lab periods. The problem is approached using the engineering design process with iterations usually necessary to arrive at the final working design. The first lab period is essentially a "brain storming" session, where students are encouraged to develop possible solutions to the proposed problem. After deciding on an appropriate design, the lab group can start constructing and testing their sensor. During the second laboratory period, the students calibrate their measurement device and demonstrate its ability to produce the correct voltage output in response to an applied force (Figure 3). For completion of the project, the lab groups submit a formal lab report, which consists of a concise problem statement, a general description of the design, detailed methods of calibration and acceptance testing, circuit schematics, and suggestions for future improvements. The report also addresses economic considerations, regulatory issues, and the societal impact of their device.

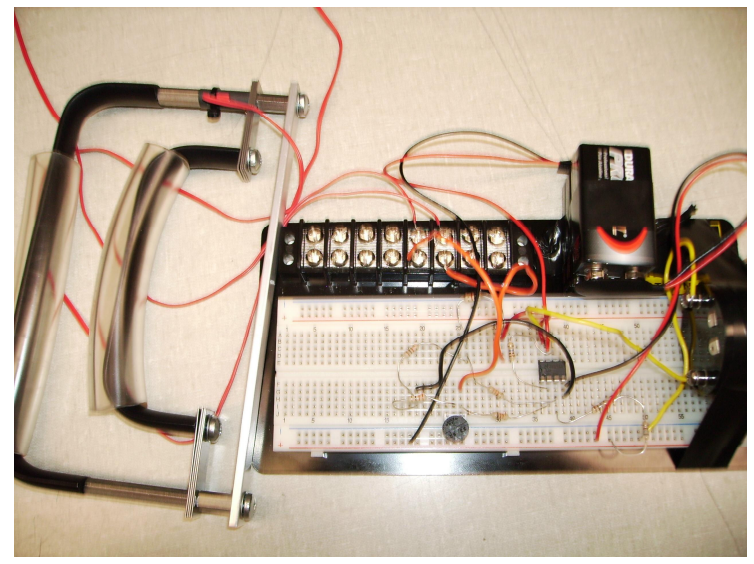

A.

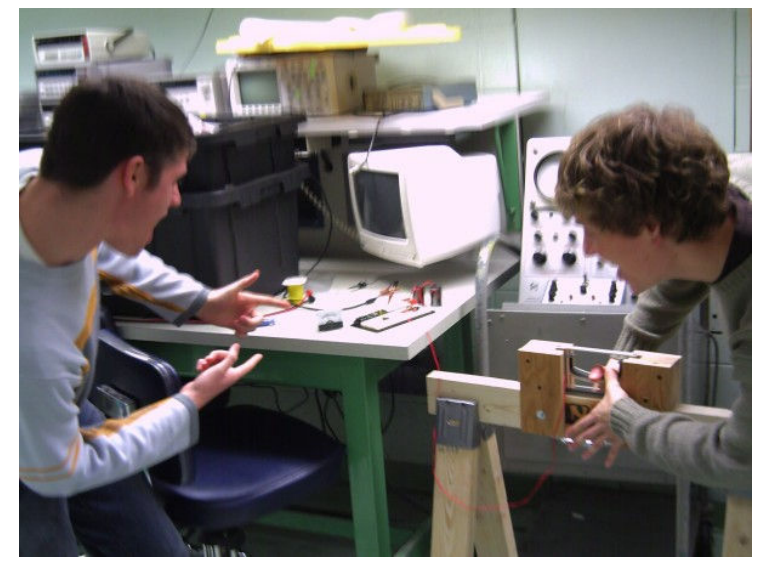

B

Figure 3. (A) Completed student dynamometer design. (B) Students demonstrate device response to applied force.

\section{Qualitative Assessment of Student Performance}

The primary goal of this course was to introduce students to bioinstrumentation circuit concepts in conjunction with measurement principles and bioelectric phenomena. To accomplish this mission and cover the topics with sufficient technical depth, the course presented a heavy workload for the students. They were exposed to many new concepts and continually pushed to apply this information before mastering the material. At nearly all of the help sessions, which were offered bi-weekly, the TA's noticed that the majority of the students came with questions pertained to both lab and lecture material. A great deal of remediation was necessary during 
office hours with students averaging more than six hours per week outside of lecture and lab to complete the pre- and post-lab exercises. Despite the rigorous workload, most of the students were able to apply the concepts as evidenced by the quality of their lab reports and the class performance on the two written exams and the lab practical. The student and faculty perception was that the second half of the course was less strenuous than the first. This was supported by the gradual reduction in number of students participating in office hours. Nevertheless, by the end of the course, the students knew how to use all of the electronic instrumentation in the lab without assistance.

Although challenging, the course effectively met its objectives. The first course objective, pertaining to the students' ability to employ circuit models and analysis techniques to understand, interpret, and predict bioelectric system behaviors, was addressed through numerous laboratory activities. Student-designed experiments to develop a circuit model of an electrolytic conducting cell and their collection and analysis of the excitable tissue stimulation data support the assertion that the first course objective was satisfied.

The second course objective involved enhancing students' experimental design, hypothesis formulation and testing, and laboratory skills. The pre-labs for almost all of the laboratories ensured that the students formulated expectations before the experiments and verified the predictions via the laboratory procedures. The experimental design process was utilized in the impedance pneumography and the nerve propagation velocity labs. Although the students did successfully complete these activities, they needed substantial assistance to achieve the desired level of competency. In the future, these labs may be modified to facilitate better learning while achieving the objectives.

The third course objective emphasizes the students' ability to design or utilize bioinstrumentation systems with adequate bandwidth, amplitude linearity, and phase linearity to faithfully record a physiological event. While these topics were implicitly presented throughout the semester, the written exams, the ECG measurement, the instrumentation amplifier design, and the design project explicitly examined the importance of satisfying the criteria for accurate measurement. The design project required the development of a device that satisfied all of the aforementioned requirements. The hand dynamometers had to respond in a timely fashion to an applied force (adequate bandwidth); the delay between the force and the output had to be consistent for any user (phase linearity); finally, and most important, the output voltage had to be linearly proportional to the force (amplitude linearity).

To quantitatively assess the students learning and retention of the course topics, the class completed a survey at the midpoint and end of the Fall 2005 semester. Students responded affirmatively to statements regarding their acquisition of skills with electrical lab measurement, instrumentation, and circuit analysis. They were particularly interested in the animal experiments. They enjoyed the hands-on experience commenting that these experiments helped validate the theoretical background information presented in lecture and lab. As expected, many students reported that the pace was too fast and it required a disproportionate amount of out-ofclass work. To partially address these concerns, the course format has been modified slightly to extend the lecture portion by $50 \%$ and reduce the pre- and post-lab assignments associated with the textbook exercises. These changes will be effective for the Fall 2006 semester. In addition, a 
follow-up survey will be administered late in the Spring 2006 semester to determine if the course prepared them for their current courses or research. Based on student feedback and a more formal course assessment, the course may be further modified for the Fall 2006 course offering.

\section{Conclusions}

The bioinstrumentation course developed at Purdue University is a novel method for integrating the teaching of bioinstrumentation from a circuit theory perspective with bioelectricity and measurement principles. The inquiry-based and hands-on laboratory exercises in conjunction with extensive pre- and post-lab assignments successfully conveyed the complex bioinstrumentation, bioelectricity and measurement concepts. Upon completion of the course, the students were able to successfully utilize circuit models of biological systems, design and conduct laboratory experiments, and design bioinstrumentation systems with adequate bandwidth, amplitude linearity, and phase linearity to faithfully record a physiological event.

\section{References}

1. Harris, T.R., J.D. Bransford, and S.P. Brophy, Roles for learning sciences and learning technologies in biomedical engineering education: a review of recent advances. Annu Rev Biomed Eng, 2002. 4: p. 29-48. 